Pacific Journal of Mathematic 


\title{
ON THE NUMBER OF PRIMITIVE PYTHAGOREAN TRIANGLES WITH AREA LESS THAN $n$
}

\author{
ROY E. WILD
}

1. Introduction. In the preceding paper Lambek and Moser have shown that if $P_{a}(n)$ is the number of primitive Pythagorean triangles with area less than $n$ then

$$
P_{a}(n)=c n^{1 / 2}+O\left(n^{1 / 3}\right),
$$

where

$$
c=\frac{\Gamma^{2}(1 / 4)}{2^{1 / 2} \pi^{5 / 2}}
$$

They conjecture that

$$
P_{a}(n)=c n^{1 / 2}-c^{\prime} n^{1 / 3}+o\left(n^{1 / 3}\right),
$$

and on the basis of a table due to Miksa they find

$$
c^{\prime} \approx .295 \text {. }
$$

Our purpose here is to show that

$$
P_{a}(n)=c n^{1 / 2}-c^{\prime} n^{1 / 3}+O\left(n^{1 / 4} \ln n\right),
$$

where

$$
c^{\prime}=-\frac{\zeta(1 / 3)\left(1+2^{-1 / 3}\right)}{\zeta(4 / 3)\left(1+4^{-1 / 3}\right)} \approx .297 .
$$

In the paper by Lambek and Moser, the problem of calculating $P_{a}(n)$ has been reduced to that of counting the number of lattice points $L(n)$ in the region $R_{1}$ defined by

Received December 3, 1953. The author is grateful to Professor E. G. Straus for calling his attention to the problem treated in this paper, and for making valuable suggestions and criticisms.

Pacific J. Math. 5 (1955), $85-91$ 


$$
x y\left(y^{2}-x^{2}\right)<n, y>x>0 .
$$

They obtain

$$
L(n)=\frac{\Gamma^{2}(1 / 4)}{2^{5 / 2} \pi^{1 / 2}} n^{1 / 2}+O\left(n^{1 / 3}\right) .
$$

We shall obtain, in place of (7),

$$
L(n)=\frac{\Gamma^{2}(1 / 4)}{2^{5 / 2} \pi^{1 / 2}} n^{1 / 2}+\left(1+2^{-1 / 3}\right) \zeta(1 / 3) n^{1 / 3}+O\left(n^{1 / 4}\right)
$$

2. Proof of $(8)$. Following is the graph of

$$
K_{1}: x y\left(y^{2}-x^{2}\right)=n \text {. }
$$

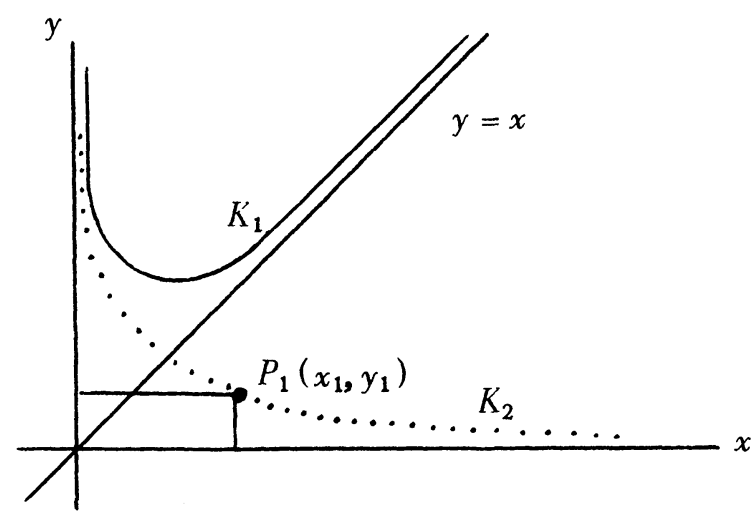

From $K_{1}$ we obtain the curve $K_{2}$ by replacing $y$ in $K_{1}$ by $y+x$ to get

$$
K_{2}: x y(x+y)(2 x+y)=n \text {. }
$$

This transformation preserves the area and number of lattice points in $R_{1}$. So we count the lattice points in $R_{2}$ defined by

$$
x y(x+y)(2 x+y)<n, x>0, y>0 .
$$

By Cardan's formulas, we obtain, from (10),

$$
x=\left(\frac{n}{4 y}\right)^{1 / 3}\left\{\left[1+\left(1-\frac{y^{8}}{108 n^{2}}\right)^{1 / 2}\right]^{1 / 3}+\left[1-\left(1-\frac{y^{8}}{108 n^{2}}\right)^{1 / 2}\right]^{1 / 3}\right\}-\frac{y}{2}
$$

and 
(13)

$$
y=\left(\frac{n}{2 x}\right)^{1 / 3}\left\{\left[1+\left(1-\frac{4 x^{8}}{27 n^{2}}\right)^{1 / 2}\right]^{1 / 3}+\left[1-\left(1-\frac{4 x^{8}}{27 n^{2}}\right)^{1 / 2}\right]^{1 / 3}\right\}-x
$$

In (13) take

$$
x=x_{1}=\left(\frac{27}{4}\right)^{1 / 8} n^{1 / 4},
$$

say, so that

$$
y=y_{1}=\left(\left(\frac{64}{3}\right)^{1 / 8}-\left(\frac{27}{4}\right)^{1 / 8}\right) n^{1 / 4}
$$

thus determining the point $p_{1}:\left(x_{1}, y_{1}\right)$ on $K_{2}$.

Let square brackets denote the greatest integer function. Then from the figure we have

$$
L(n)=\sum_{x=1}^{\left[x_{1}\right]}[y]+\sum_{y=1}^{\left[y_{1}\right]}[x]-\left[x_{1}\right]\left[y_{1}\right]-l(n)
$$

where $l(n)$ is the number of lattice points on $K_{2}$. Now $l(n)$ is zero unless $n$ is an integer $N$. For nonintegral $n$ we can prove (8). For small positive $\epsilon$ we obtain (8) for, say, $N+\epsilon$ and $N-\epsilon$, so that trivially

$$
l(n)=O\left(n^{1 / 4}\right) \text { for all real } n .
$$

By definition,

$$
\left[x_{1}\right]=O\left(n^{1 / 4}\right),\left[y_{1}\right]=O\left(n^{1 / 4}\right),
$$

so that we may drop the brackets in (14) with an error $O\left(n^{1 / 4}\right)$. Then, by use of (15) and (16), (14) becomes

$$
L(n)=\sum_{x=1}^{\left[x_{1}\right]} y+\sum_{y=1}^{\left[y_{1}\right]} x-x_{1} y_{1}+O\left(n^{1 / 4}\right) .
$$

We shall estimate the above sums by the Euler-Maclaurin summation formula in the form: 
(18) $\sum_{k=a}^{b} f(k)=\int_{a}^{b} f(x) d x+\frac{1}{2} f(b)+\frac{1}{2} f(a)+\int_{a}^{b}\left(x-[x]-\frac{1}{2}\right) f^{\prime}(x) d x$.

We obtain from ( 17 ):

(19)

$$
\begin{aligned}
L(n) & =\int_{1}^{\left[x_{1}\right]} y d x+\int_{1}^{\left[y_{1}\right]} x d y-x_{1} y_{1}+O\left(n^{1 / 4}\right) \\
& +\frac{1}{2} y\left(\left[x_{1}\right]\right)+\frac{1}{2} y(1)+\frac{1}{2} x\left(\left[y_{1}\right]\right)+\frac{1}{2} x(1) \\
& +\int_{1}^{\left[x_{1}\right]}\left(x-[x]-\frac{1}{2}\right) \frac{d y}{d x} d x+\int_{1}^{\left[y_{1}\right]}\left(y-[y]-\frac{1}{2}\right) \frac{d x}{d y} d y .
\end{aligned}
$$

In the first two terms of (19), we may drop brackets with an error of $O\left(n^{1 / 4}\right)$, so that, if $A$ represents the entire area of $R_{2}$, we may replace the first three terms of (19) by

$$
A-\int_{0}^{1} y d x-\int_{0}^{1} x d y+O\left(n^{1 / 4}\right)
$$

Now from ( 12 ) and (13) we have

$$
\begin{aligned}
x & =(n / 4 y)^{1 / 3}\left(2^{1 / 3}+O\left(y^{8 / 3} / n^{2 / 3}\right)\right)+O(y) \\
& =(n / 2 y)^{1 / 3}+O\left(y^{7 / 3} / n^{1 / 3}\right)+O(y),
\end{aligned}
$$

and similarly

$$
y=(n / x)^{1 / 3}+O\left(x^{7 / 3 / n^{1 / 3}}\right)+O(x) .
$$

Substituting in (20), we obtain

$$
\begin{aligned}
A-3 n^{1 / 3} / 2+O\left(n^{-1 / 3}\right)+O(1) & \\
-3 n^{1 / 3} / 2^{4 / 3}+O\left(n^{-1 / 3}\right) & +O(1)+O\left(n^{1 / 4}\right) \\
& =A-\frac{3}{2}\left(1+2^{-1 / 3}\right) n^{1 / 3}+O\left(n^{1 / 4}\right) .
\end{aligned}
$$

The fifth and seventh terms of (19) are $O\left(n^{1 / 4}\right)$. Also 


$$
\frac{1}{2} y(1)=\frac{1}{2} n^{1 / 3}+O(1) \text { and } \frac{1}{2} x(1)=n^{1 / 3 / 2^{4 / 3}}+O(1) \text {. }
$$

Differentiating the expansions of $x$ and $y$ in (21) and (22) we obtain

$$
\begin{aligned}
& d x / d y=-n^{1 / 3} y^{-4 / 3} / 3 \cdot 2^{1 / 3}+O\left(y^{4 / 3} / n^{1 / 3}\right)+O(1), \\
& d y / d x=-n^{1 / 3} x^{-4 / 3} / 3+O\left(x^{4 / 3} / n^{1 / 3}\right)+O(1) .
\end{aligned}
$$

We now rewrite the last two terms of (19) as

$$
\begin{aligned}
& \int_{1}^{n^{1 / 8}}\left(x-[x]-\frac{1}{2}\right) \frac{d y}{d x} d x+\int_{n^{1 / 8}}^{\left[x_{1}\right]}\left(x-[x]-\frac{1}{2}\right) \frac{d y}{d x} d x \\
& +\int_{1}^{n^{1 / 8}}\left(y-[y]-\frac{1}{2}\right) \frac{d x}{d y} d y+\int_{n^{1 / 8}}^{\left[y_{1}\right]}\left(y-[y]-\frac{1}{2}\right) \frac{d x}{d y} d y .
\end{aligned}
$$

Since $|d y / d x|$ is monotonic decreasing, we have, by the second mean value theorem for integrals, and (25),

$$
\begin{aligned}
\int_{n^{1 / 8}}^{\left[x_{1}\right]}\left(x-[x]-\frac{1}{2}\right) \frac{d y}{d x} d x & =\left.\frac{d y}{d x}\right|_{x=n^{1 / 8}} \int_{n^{1 / 8}}^{h}\left(x-[x]-\frac{1}{2}\right) d x \\
& =O\left(n^{1 / 6}\right) O(1)=O\left(n^{1 / 6}\right) .
\end{aligned}
$$

Similarly

$$
\int_{n}^{\left[y_{1}\right]}\left(y-[y]-\frac{1}{2}\right) \frac{d x}{d y} d y=O\left(n^{1 / 6}\right) .
$$

Substituting (24) and (25) in the remaining terms of (26) yields

$$
\text { (29) } \begin{aligned}
& \int_{1}^{n^{1 / 8}}\left(x-[x]-\frac{1}{2}\right) \frac{d y}{d x} d x=-\frac{n^{1 / 3}}{3} \int_{1}^{n^{1 / 8}}\left(x-[x]-\frac{1}{2}\right) x^{-4 / 3} d x+O(1) \\
& =-\frac{n^{1 / 3}}{3} \int_{1}^{\infty}\left(x-[x]-\frac{1}{2}\right) x^{-4 / 3} d x+\frac{n^{1 / 3}}{3} \int_{n^{1 / 8}}^{\infty}\left(x-[x]-\frac{1}{2}\right) x^{-4 / 3} d x+O(1) \\
& =-\frac{n^{1 / 3}}{3} \int_{1}^{\infty}\left(x-[x]-\frac{1}{2}\right) x^{-4 / 3} d x+O\left(n^{1 / 6}\right),
\end{aligned}
$$


and simi larly

$$
\begin{aligned}
\int_{1}^{n^{1 / 8}}\left(y-[y]-\frac{1}{2}\right) \frac{d x}{d y} d y & =-\frac{n^{1 / 3}}{3 \cdot 2^{1 / 3}} \int_{1}^{\infty}\left(y-[y]-\frac{1}{2}\right) y^{-4 / 3} d y \\
& +O\left(n^{1 / 6}\right) .
\end{aligned}
$$

Collecting the preceding results, we have

$$
\begin{aligned}
L(n) & =A-\frac{3}{2}\left(1+2^{-1 / 3}\right) n^{1 / 3}+O\left(n^{1 / 4}\right)+O\left(n^{1 / 4}\right)+n^{1 / 3} / 2+O(1) \\
& +n^{1 / 3} / 2^{4 / 3}+O(1)+\left(1+2^{-1 / 3}\right) c_{1} n^{1 / 3}+O\left(n^{1 / 6}\right) \\
& =A-\left(1+2^{-1 / 3}\right)\left(1-c_{1}\right) n^{1 / 3}+O\left(n^{1 / 4}\right),
\end{aligned}
$$

where

$$
c_{1}=\int_{1}^{\infty}\left(x-[x]-\frac{1}{2}\right) d x^{-1 / 3}=\zeta(1 / 3)+1
$$

Now $A$ is the area of $R_{2}$ and therefore the area of $R_{1}$. Its value as calculated by Lambek and Moser is

$$
A=c_{2} n^{1 / 2}, \quad c_{2}=\frac{\Gamma^{2}(1 / 4)}{2^{5 / 2} \pi^{1 / 2}}
$$

Substitution from (32) and (33) in (31) yields (8).

3. Derivation of (4). Let $c_{3}=-\left(1+2^{-1 / 3}\right) \zeta(1 / 3)$, so that $(31)$ becomes

$$
L(n)=c_{2} n^{1 / 2}-c_{3} n^{1 / 3}+O\left(n^{1 / 4}\right) .
$$

Following the notation of Lambek and Moser, we can write (34) as

$$
L(R t)=c_{2} t^{2}-c_{3} t^{4 / 3}+O(t)
$$

From their equation (14) we have

$$
L^{\prime}(R t)=\sum_{i \geq 1} \mu(i)\left\{c_{2} \frac{t^{2}}{i^{2}}-c_{3} \frac{t^{4 / 3}}{i^{4 / 3}}+O(t / i)\right\}
$$




$$
\begin{aligned}
& =\left\{6 c_{2} / \pi^{2}+O(1 / t)\right\} t^{2}-\left\{c_{3} / \zeta(4 / 3)+O\left(1 / t^{1 / 3}\right)\right\} t^{4 / 3}+O(t \ln t) \\
& =\frac{6 c_{2}}{\pi^{2}} t^{2}-\frac{c_{3}}{\zeta(4 / 3)} t^{4 / 3}+O(t \ln t) .
\end{aligned}
$$

Then from their equations (6), (15), and our (36), we have

$$
\begin{aligned}
& P_{a}(n)= \sum_{i \geq 0}(-1)^{i}\left\{\frac{6 c_{2} n^{1 / 2}}{\pi^{2} 2^{i}}-\frac{c_{3} n^{1 / 3}}{\zeta(4 / 3) 4^{i / 3}}\right. \\
&\left.+O\left(\frac{n^{1 / 4}}{4^{i / 4}} \ln \frac{n}{4^{i}}\right)\right\} \\
&= 4 c_{2} n^{1 / 2 / \pi^{2}}-\frac{c_{3} n^{1 / 3}}{\zeta(4 / 3)\left(1+4^{-1 / 3}\right)}+O\left(n^{1 / 4} \ln n\right) \\
&=c n^{1 / 2}-c^{\prime} n^{1 / 3}+O\left(n^{1 / 4} \ln n\right) .
\end{aligned}
$$

This is (4).

UNIVERSITY OF IDAHO 




\section{Pacific Journal of Mathematics}

\section{Vol. 5, No. $1 \quad$ September, 1955}

Frank Herbert Brownell, III, Flows and noncommuting projections on

Hilbert space ................................... 1

H. E. Chrestenson, A class of generalized Walsh functions ............. 17

Jean Bronfenbrenner Crockett and Herman Chernoff, Gradient methods of maximization ................................... 33

Nathan Jacob Fine, On groups of orthonormal functions. I .......... 51

Nathan Jacob Fine, On groups of orthonormal functions. II ............ 61

Frederick William Gehring, A note on a paper by L. C. Young .......... 67

Joachim Lambek and Leo Moser, On the distribution of Pythagorean

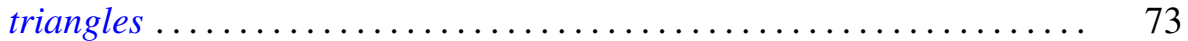

Roy Edwin Wild, On the number of primitive Pythagorean triangles with

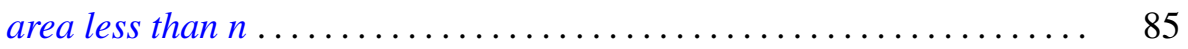

R. Sherman Lehman, Approximation of improper integrals by sums over multiples of irrational numbers ........................ 93

Emma Lehmer, On the number of solutions of $u^{k}+D \equiv w^{2}(\bmod p) \ldots 103$

Robert Delmer Stalley, A modified Schnirelmann density............... 119

Richard Allan Moore, The behavior of solutions of a linear differential equation of second order............................. 125

William M. Whyburn, A nonlinear boundary value problem for second order differential systems. 